\title{
GPPS-BJ-2019-0233
}

\section{Flow Control of Radial Migration Along the Rotor Suction Surface for Low-speed Ducted Fan}

\author{
Yuchen Dai \\ Aeroengine Simulation Research Center, \\ School of Energy and Power Engineering, \\ Beihang University \\ sy1804201@buaa.edu.cn \\ Beijing,China
}

\author{
Donghai Jin \\ Aeroengine Simulation Research Center, \\ School of Energy and Power Engineering, \\ Beihang University \\ jdh@buaa.edu.cn \\ Beijing,China
}

\author{
Xingmin Gui \\ Aeroengine Simulation \\ Research Center, School of \\ Energy and Power \\ Engineering, Beihang \\ University \\ guixm@buaa.edu.cn \\ Beijing,China
}

\author{
Wei Xiao \\ Aeroengine Simulation \\ Research Center, School of \\ Energy and Power \\ Engineering, Beihang \\ University \\ xiaovex@yeah.net
}

\begin{abstract}
In order to suppress the radial migration of the suction surface of the rotor blade. Based on a preliminary design of the ducted fan, the control of the radial migration is realized by changing the rotor sweep type, the load distribution along the span and the chord direction. The results show that for the over-bending blade, when the load of root is increased, the static pressure rise coefficient and the diffuser load of root is reduced, so the root radial migration flow is weakened. When the load along the chord direction is moved forward, the trailing load is reduced, the pressure difference at the suction and pressure surface of trailing edge is reduced, and the tendency of the pressure surface airflow flow around the trailing edge to the suction surface is weakened, the recirculation zone is eliminated, so the radial migration is weakened. When the blade is swept forward, the radial pressure gradient is increased, and the radial migration of the low-energy fluid in the boundary layer is suppressed. The optimized ducted fan has a higher pressure ratio, efficiency and margin than the prototype fan.
\end{abstract}

\section{INTRODUCTION}

High efficiency has always been one of the goals pursued by aircraft engine compressors. However, for turbomachinery, due to the presence of viscosity, not only the wake vortex is formed at the blade exit, but also the viscous boundary layer exists on both the blade surface and the end wall.There is a strong interaction between them and the main flow, resulting in a so-called secondary flow. The secondary flow is the main source of the increase of the mechanical loss and the decrease of the efficiency of turbomachinery. In the modern compressor, the loss caused by the secondary flow can be equivalent to or exceed the two-dimensional flow loss of the cascade. Therefore, it is necessary to conduct an in-depth and comprehensive investigation of the secondary flow in the compressor.

Several studies have been published over the years in the open literature regarding the effects of sweeping on the secondary flow loss. Yamaguchi et al.[1] believe that in conventional rotor blades, due to the unbalance between centrifugal force and radial pressure gradient, radial migration from the root to the tip of the blade boundary layer occurs,so low energy fluid stacks near the casing,which will 
increase the aerodynamic loss and also reduce the stall margin; and when the rotor blade is swept forward, the radial migration fluid will fall off the blade surface with the wake vortex before reaching the blade tip. At the same time, since the airflow at the tip zone of the blade first enters the blade, the high pressure region after the minimum static pressure of the suction surface is closer to the leading edge than the blade root, so the pressure at the tip of the blade is greater than that of root at the same chord location, and the low energy fluid of radial migration is suppressed. Krishnakumar[2] think for the tip forward-swept rotor, the leading edge of the tip touches the fluid earlier,so the energy conversion causes the pressure of tip to rise, changing the original radial pressure gradient, causing the fluid to move downward to match the new pressure gradient.Compared with Yamaguchi's explanation, Krishnakumar does not limit the influence of the sweep to radial migration in the boundary layer, Krishnakumar think that it can be seen as the effect of on the mainstream radial balance.In short, the sweeping blades play an important role in the flow control. By correctly understanding the flow field and adopting a reasonable form of sweeping, the secondary flow loss can be reduced and better characteristics can be obtained.

The current study examined the effect of loading distributions on the stage performance However,these studies are based on the turbine cascade and the conclusions of these studies are often contradictory.Patterson and Hoeger[3] studied the effect of load distributions on the performance of three turbine vanes under low transonic Mach numbers. They concluded that the aft-loaded profile yielded the worst performance for the conditions investigated.The performance of the first and second stage stator vanes of an low pressure turbine were tested for both front and aft-loaded profiles by Hashimoto and Kimura[4].It was found that the front-loaded designs yielded lower losses and a wider range of usable incidence for high subsonic Mach numbers.Hoheisel et al.[5] studied a front-loaded profile together with two aft-loaded ones.The three designs had the same overall aerodynamic loading.Tests performed at low Mach numbers showed that lower losses can be obtained with an aft-loaded pressure distribution. The influences of blade loading profile on wake convection and wake/wake interaction were studied in two different blade designs for high-pressure turbines(frontloaded and aft-loaded),installed in linear cascades from Benjamin T.Luymes et al[6].The turbulence kinetic energy in the wake was approximately $30 \%$ higher for the front-loaded blade when the wake entered the measurement field-ofview,this means that the front-loaded blade obtain higher losses.The research on the load distribution in the compressor is mainly focused on the study of the tip leakage flow.Jürgen Hübner and Leonhard Fottner[7] think that the strength of tip leakage flow loss is controlled by the blade loading.Changes in loss generation associated with altering rotor tip blade loading of an embedded rotor-stator compressor stage are assessed at Aniwat Tiralap ea al.[8] paper.Aft-loading rotor blade tip delays the formation of tip leakage flow,resulting in a relatively less mixed-out tip leakage flow at the rotor outlet and a reduction in overall tip leakage mass flow, hence a lower loss.However,the attendant changes in tip flow angle distribution are such that there is an overall increase in the flow angle mismatch between tip flow and main flow,leading to higher loss generation. The latter outweights the former.

The current researchs are mainly concentrated on the impact of load distributions to turbine cascades, while the research in compressors is mainly on the influences to the tip leakage flow. There are few studies about the impact to other secondary flow, especially the radial migration flow. Therefore, this paper mainly studies the influence of the chordwise load distribution and the radial load distribution on the radial migration flow of the rotor suction surface, and also explores the sweeping influence to radial migration flow.

\section{METHODOLOGY}

\section{Introduction about numerical simulations and prototype fan}

The compressor stage used to assess the performance variation was taken from a subsonic ducted fan.The geometrical parameters and operating conditions are provided in Table 1.

\begin{tabular}{lr}
\hline Parameters & Values \\
\hline Rotor tip-to-hub ratio & 0.28 \\
Rotor solidity & 1.35 \\
Rotor aspect ratio & 2.28 \\
Reynolds number & $1 \times 10^{6}$ \\
Inlet Mach number & 0.35 \\
Rotor tip clearance size & $1 \%$ span \\
Rotating speed(r/min) & 2500 \\
Tip radius(m) & 0.53 \\
\hline
\end{tabular}

Table1 General characteristic of the compressor stage

A commercial CFD solver,NUMECA, has been chosen for computing flow fields of the selected compressor stage. A series of URANS computations has been performed with double precision,second-order advection scheme,secondorder backward(implicit) Euler time-differencing scheme,and first-order turbulence numeric.The k- $\omega$ shear stress transport turbulence model has been employed as the turbulence model.The working fluid is treated as an ideal gas with constant $C_{p}$. The computational geometry consists of one upstream rotor and one downstream stator blade(single passage).The mesh resolution of the stage is $1.12 \times 10^{6}$ nodes. Wall function is utilized with $y^{+}$of approximately 10 on the blade surface to reduce required computational resource.The boundary condition at the stage inlet is specified as a radial distribution of stagnation pressure,stagnation temperature and flow angle.The boundary condition at the stage outlet is specified as a radial distribution of circumferentially averaged static pressure, which can be varied to obtain a desired operating condition for different blade designs.

The computatioanal setup used has been assessed against those in the literature.Numeca has been has been demonstrated to be capable of providing accurate 
assessments of overall performance of a multistage compressor with a single passage steady analysis[9].Similary,Numeca was used by Eric A.Crosh ea al[10] to investigate the effect of rotor shroud(casing) out-ofroundness on the unsteady pressure loading for the blade row of a full-stage turbine. Laroslav Druzhinin ea al[11] found that the flow field of the investigated high bypass fan,based on Numeca,is similar to the experimental result.Grid sensitivity analysis carried out based on steady computations has shown insignificant changes in numerical dissipation of the new blade designs relative to the original one.These assessments ensure the capability of Numeca in computing the flow field of axial compressors and appropriateness of the computational setup required to answer the research questions.

The results obtained by numerical simulation are as follows:

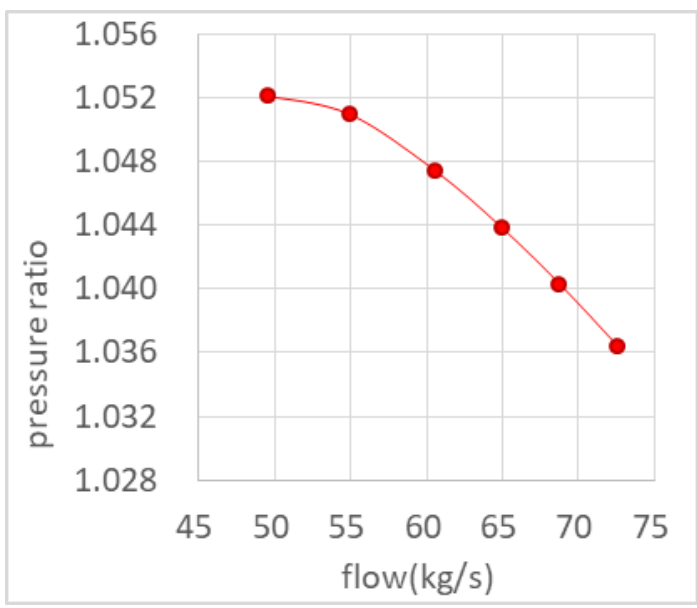

Figure1 pressure ratio characteristic

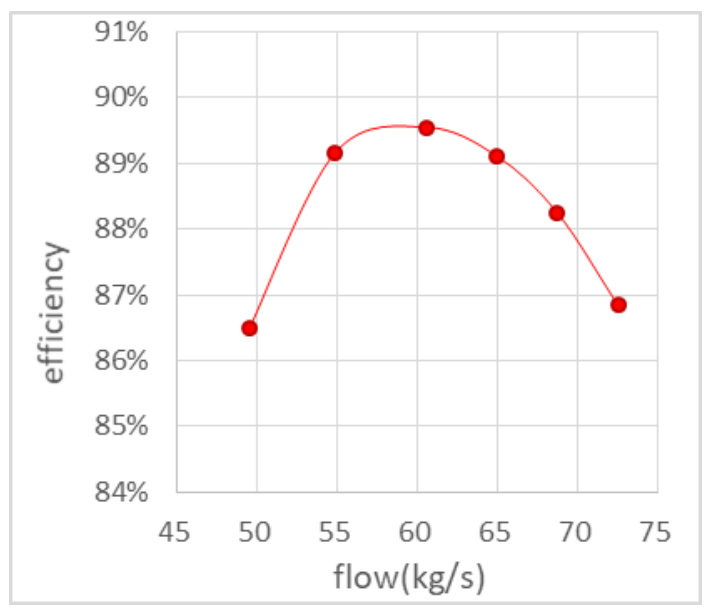

Figure2 efficiency characteristic

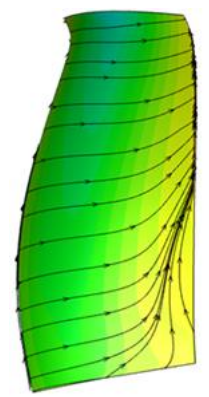

Figure3 Limiting streamline of suction surface

Figure 1 and Figure 2 show the pressure ratio characteristics and efficiency characteristics of the fan, and Figure 3 shows the limiting streamline of rotor and stator suction surfaces. It can be seen from the figure that there is a significant radial migration of the rotor. The following three methods will be used to optimize the radial migration of the rotor surface and explore the relevant flow mechanism.

\section{The optimization of load distribution along span direction}

The typical load distribution rules of fan design include equal load distribution, equal reaction degree distribution, exponential distribution and variable distribution along the span direction. In this paper, the influence of the distribution of the rotor load along the span direction on the fan performance is studied. According to the analysis results, the appropriate load distribution is selected to optimize the fan. In the preliminary design, the variable load distribution is selected. In order to study the influence of the different distribution modes of load on the design results, a variety of fans with different load distributions are designed. Three representative distributions are used in this paper. The three load distributions are shown in Fig. 4. A is the distribution used in the preliminary design, $\mathrm{C}$ is the equal load distribution, $\mathrm{B}$ is the linear distribution from root to tip and the amount of load is between A and C. For the convenience of description, the rotors of the three distributions $\mathrm{A}, \mathrm{B}$, and $\mathrm{C}$ are respectively referred to as $\mathrm{A}, \mathrm{B}, \mathrm{C}$ rotor.

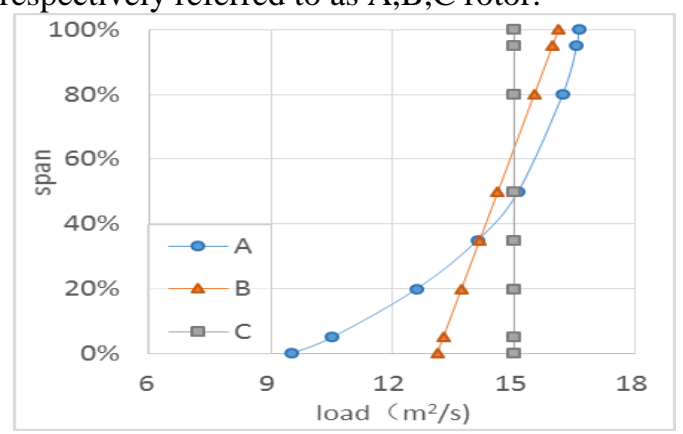

Figure 4 Three load distribution

Numerical calculations are made for the ducted fans of three different load distribution designs. Figure 5 is a comparison of the limiting streamline of suction surface of three different load distribution along the span direction. It can be seen from the figure that the difference of the limiting streamline is not obvious above the middle span of the blade. The migration trend of the $\mathrm{B}$ and $\mathrm{C}$ rotors is slightly lower than that of the A rotor. The influence of load distribution to the blade tip migration is not obvious. The radial migration flow of the root is obviously different. With the design load of the root gradually increasing, the radial migration trend of the root is gradually slowed down. In the A rotor, the lowenergy fluid in the boundary layer of the suction surface of the blade root migrates along the radial direction due to the centrifugal force, and develops almost straight to the middle span; In the B rotor, the slope of the limiting streamline generated from the root is reduced, indicating that the tendency of the low energy fluid flowing to the middle span 
is weakened; In the $\mathrm{C}$ rotor, as the amount of root load increases further, the streamline in the $10 \%$ span of the root directly flows out from the trailing edge of the blade and no longer migrates to the middle span, indicating that the radial migration in this zone is basically suppressed.

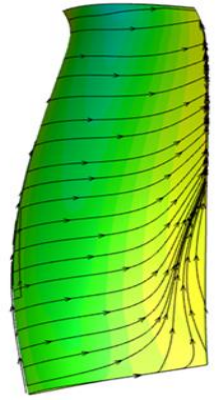

A

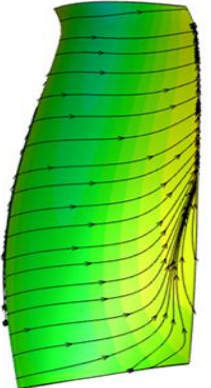

B

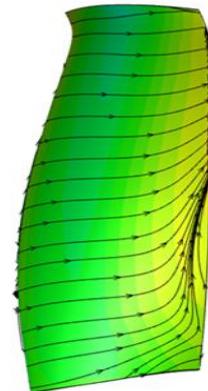

C
Pressure: $86000 \quad 90000 \quad 94000 \quad 98000 \quad 102000 \quad 106000$

\section{Figure 5 Limiting streamline of suction surface}

In general, reducing the amount of root load helps to improve the blade corner zone flow. However, the calculation results in this paper are different. The calculations show that the increase of root load suppress the root migration flow. The main reason is that the root diameter of the ducted fan of this paper is small, and the blade root of three different load distribution has been overbending(over-bending means that the rotor outlet flow angle is positive, The positive and negative signs are shown in Figure 9).The increase of the root load increases turning angle, but the static pressure rise coefficient decreases, the diffuser load decreases, and the radial pressure gradient also changes. The following is a detailed analysis.

Figure 6 is a schematic diagram of the compressor cascade. Since the cascade is an expansion passage, the static pressure rises with the increase of the area, and the static pressure rise can be evaluated by the static pressure rise coefficient. The higher the static pressure rise coefficient, The more easily the flow is separated, the static pressure rise coefficient is defined as:

$$
C_{p}=\frac{p_{2}-p_{1}}{0.5 \cdot \rho \cdot w_{1}^{2}}
$$

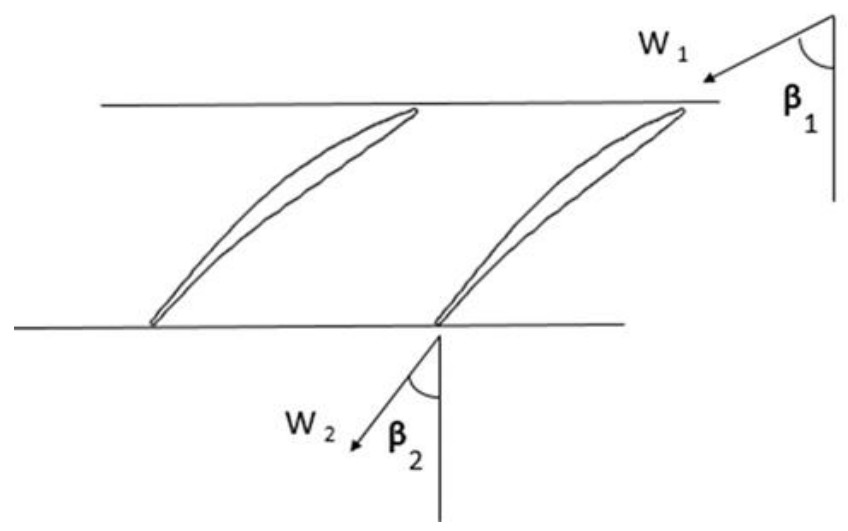

Figure 6 compressor cascade

For incompressible flow, and without considering the change in the tangential velocity of the inlet and outlet, in the ideal case, derived from the Bernoulli equation and the continuous equation:

$$
C_{p}=1-\frac{\left(\cos \beta_{1}\right)^{2}}{\left(\cos \beta_{2}\right)^{2}}
$$

Among them, $\beta_{1} 、 \beta_{2}$ are the inlet and outlet airflow angles respectively, and the metal angle can replace the airflow angle without considering the incidemce angle and the deviation angle for qualitative analysis. Figure 7 and Figure 8 are the comparison of the metal angles of the inlet and outlet of the three rotors A, B and C. For rotors with different load distribution designs, the inlet metal angle remains basically the same at the same span, and the outlet metal angle decreases with increasing load. In the rotor A, increasing the root load will reduce the exit metal angle, so that the calculated static pressure rise coefficient will increased.the A-rotor has been over-bending near the root part, and the exit metal angle is negative. As the amount of root load increases, the exit metal angle decreases, but its absolute value increases. From Equation 2, the static pressure rise coefficient of the blade root is reduced. The numerical results reflect well the static pressure changes in the root region with different load distribution. From the static pressure cloud diagram of the rotor suction surface in Figure 5 , it can be seen that the static pressure of the $\mathrm{C}$ rotor is significantly smaller than $\mathrm{A}$ in the root region. The radial pressure gradient of the boundary layer fluid in the blade root region is increased, which helps overcome the centrifugal force,so the radial migration of the root of the $\mathrm{C}$ rotor is weaker than that of A. If the root is not over-bending, then reducing the root load can simultaneously reduce the root load factor and the static pressure rise coefficient. For such a rotor, improving the root load is beneficial to improve the flow in the corner zone.

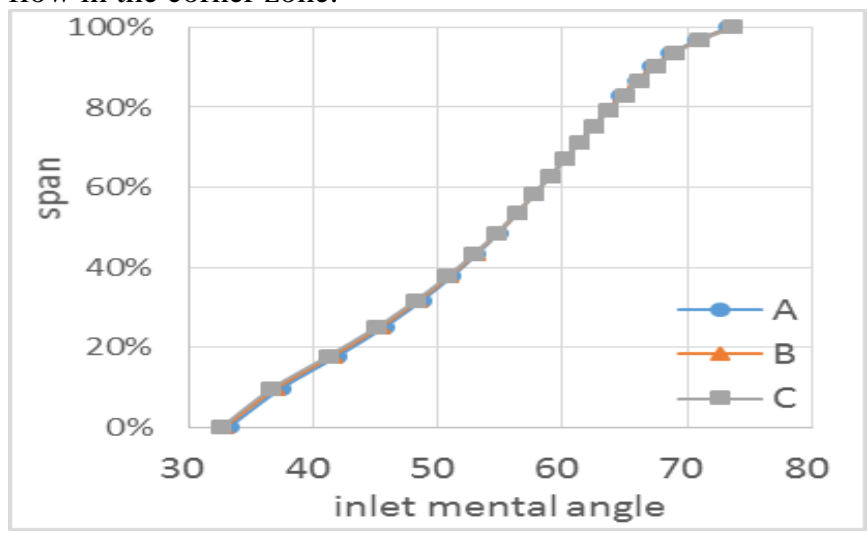

Figure 7 inlet mental angle of rotor 


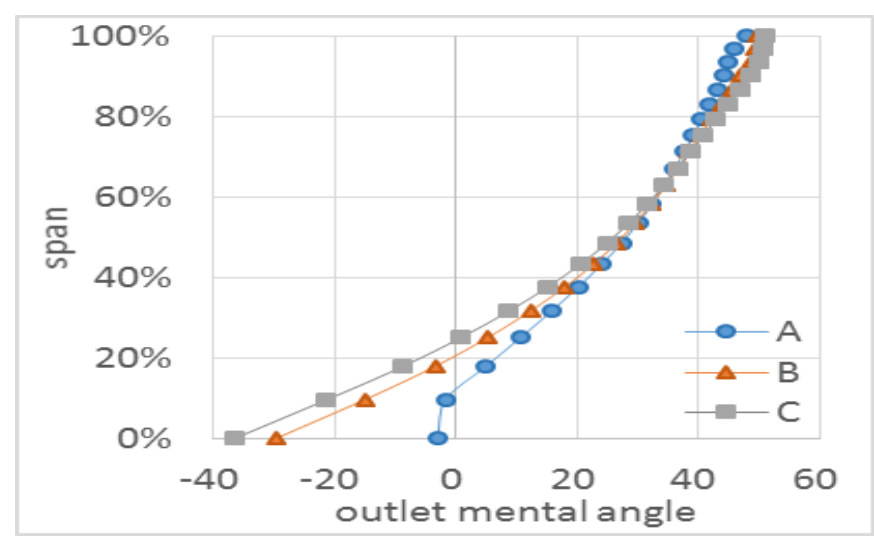

Figure 8 outlet mental angle

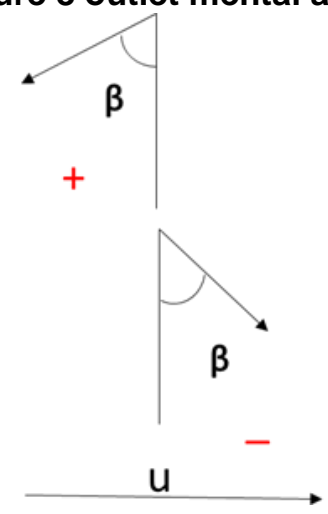

Figure 9 positive and negative mental angle sign

Rotor A is the prototype compressor. It can be seen from the above three schemes that the $\mathrm{C}$ scheme is the best solution, that is, the increase of the root load can suppress the root radial migration flow for the over-bending rotor studied in this paper. For the convenience of subsequent further comparisons, the $\mathrm{C}$ scheme is referred to as DF-2.

\section{The optimization of load distribution along chord direction}

In order to further control the migration flow of the rotor suction surface, the load distribution along the chord direction is optimized on the basis of DF-2, and the optimized scheme is recorded as DF-3.

The DF-3 mainly adjusts the distribution of the 20\%$50 \%$ span along the chord direction, and the 5\% span is slightly adjusted, while the upper part of the blade remains unchanged. Figure 10 and Figure 11 are the load distribution comparison of the $20 \%$ and $50 \%$ span along chord distribution.Comparing with DF-2 scheme, The front-loading of the DF-3 scheme is strengthened, the adjustment of $20 \%$ span is slightly larger than the $50 \%$ span. Fig. 12 is a comparison of the maximum deflection position of the blade along the span, and the maximum deflection position is significantly moved forward at the $20 \%-50 \%$ span.

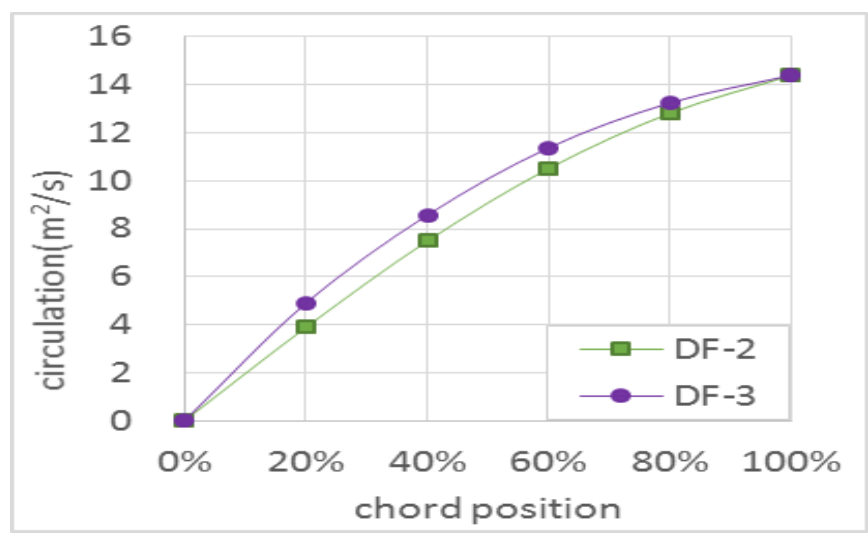

Figure $1020 \%$ span load distributon

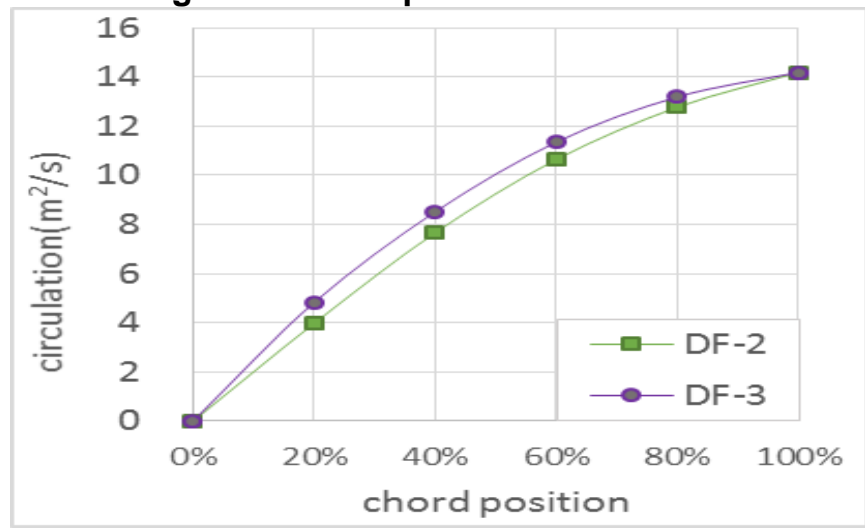

Figure $1150 \%$ span load distribution

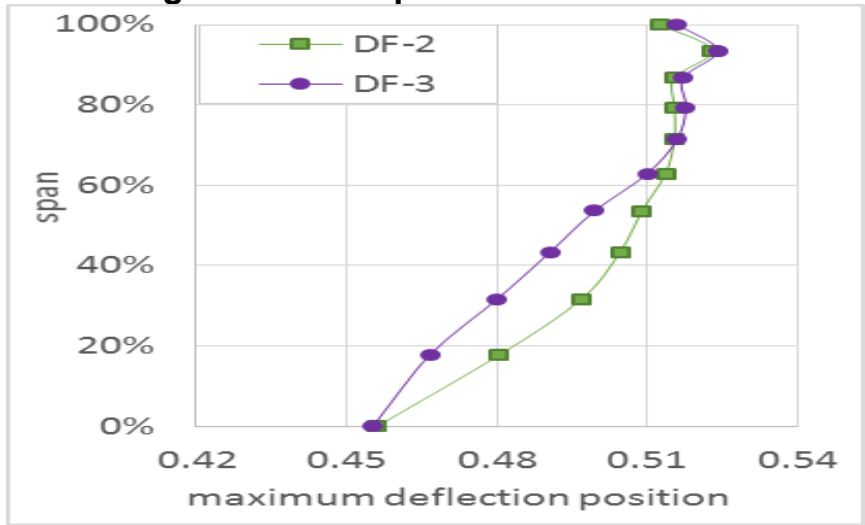

Figure 12 maximum deflection position

Figure 13 and Figure 14 show the comparison of the characteristics of the DF-2 and DF-3 fans obtained by numerical simulation.The three-dimensional numerical results show that the pressure ratio and efficiency of the fan near the design point increase after the load of the rotor blade is move forward. Mainly because the front load type can achieve a higher pressure ratio, and the efficiency is determined by the loss and the effective work. The increase of the pressure ratio means that the effective work increases, and the efficiency is significantly increased without deterioration of the flow field. Figure 15 and Figure 16 show the comparison of the total pressure ratio and efficiency of the rotor near the design point along the span distribution. The increase of the pressure ratio is mainly concentrated in the range of $20 \%-50 \%$ and the efficiency is improved in the $10-40 \%$ range. 


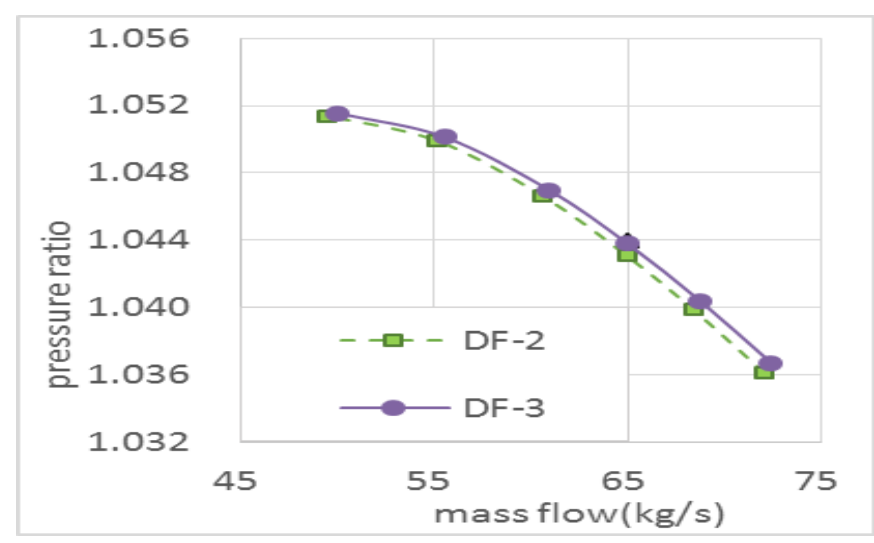

Figure 13 pressure ratio characteristics

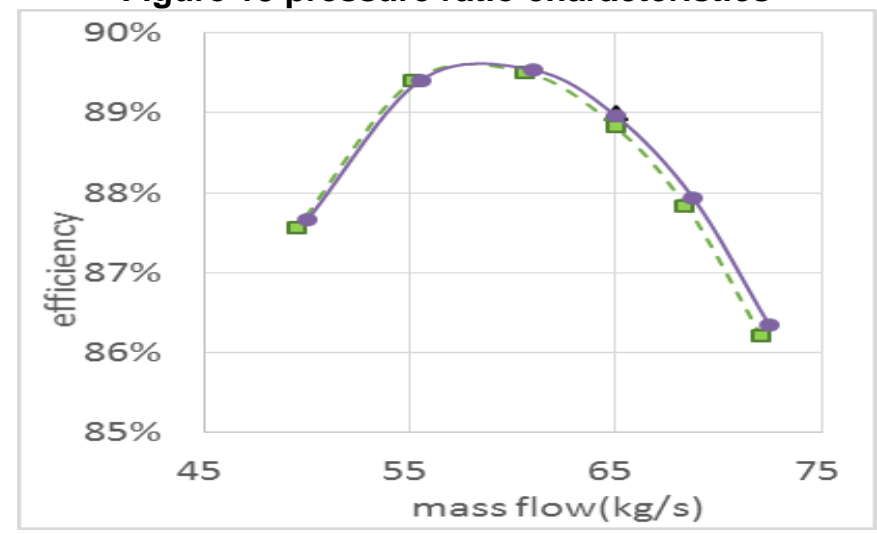

\section{Figure 14 efficiency characteristics}

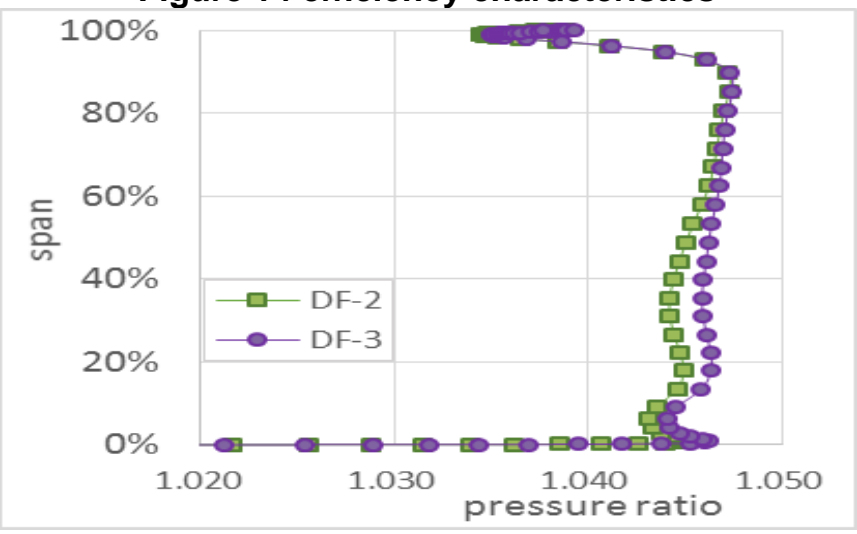

Figure 15 pressure ratio along the span direction

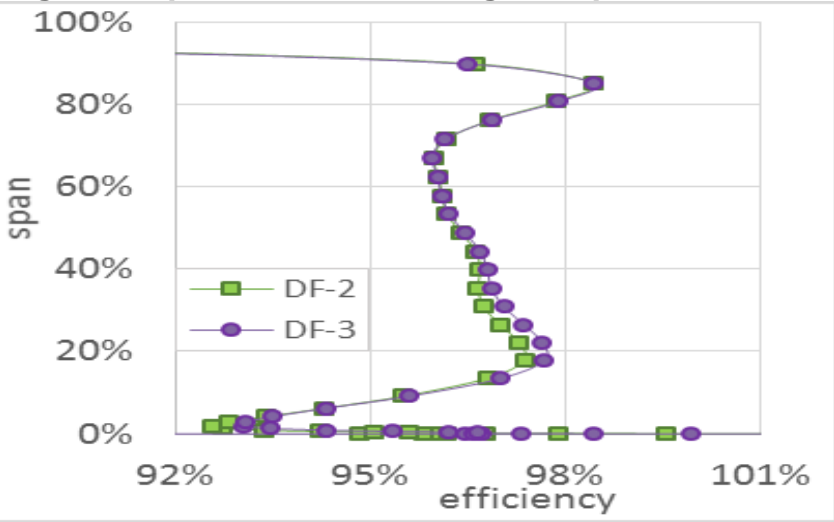

Figure 16 efficiency along the span direction

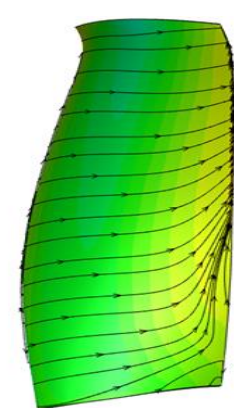

$\mathrm{DF}-2$

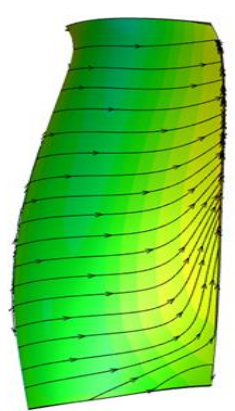

DF-3

\section{Figure 17 limiting streamline of rotor suction surface}

Figure 16 is a comparison of the limiting streamline of the suction surface of the rotor. It can be seen from the figure that the radial migration of the suction surface is controlled, and in the range of $20 \%-50 \%$ span, the backward flow zone disappears and the chord area affected by the radial migration decreases. In the previous discussion of the distribution of load distribution, it was found that reducing the range of 20\%-50\% span of the load helps to suppress the radial migration of the area, and in this section, increasing the load will increase the pressure and work of the area, but the radial migration of the region is significantly inhibited. For further explanation, comparing the surface static pressure distribution of 30\% span, as shown in Fig. 18, the pressure gradient between the suction surface and the pressure surface is larger in the DF-2 scheme than DF-3 at the leading edge, while near the trailing edge, that is larger at DF-3 scheme, indicating that the load at the leading edge of the blade is increased. The article believes that it is precisely because the pressure gradient between suction surface and pressure surface at the trailing edge of the blade is reduced, the flow from the pressure surface to the suction surface at the trailing edge is weakened, the backflow zone is eliminated, and the radial migration is weakened.

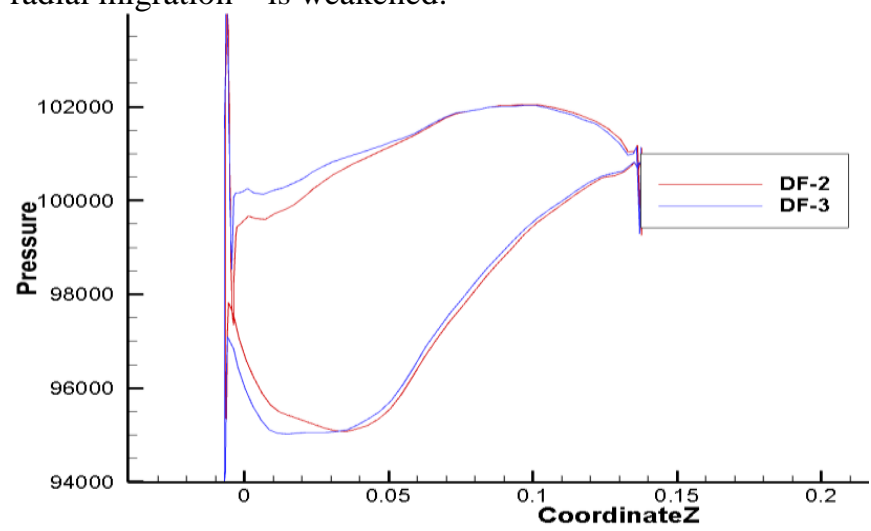

Figure 18 static pressure curve of blade surface at $30 \%$ span

\section{The optimization of rotor sweep type}

Since there is no shock wave in the low speed fan, the forward swept blade is generally used in the low speed fan rotor to control the radial migration flow of the suction 
surface of the rotor and to change the incidence angle of the tip, improve the stall margin, and suppress the tip leakage flow. It also acts to reduce aerodynamic noise. This section has optimized the sweep type, and the rotor blade is swept forward on the basis of the DF-3. The new scheme is DF-4. The comparison of the meridional channel of the forwardswept fan is shown in Figure 19. The DF-4 fan rotor has a forward sweep.

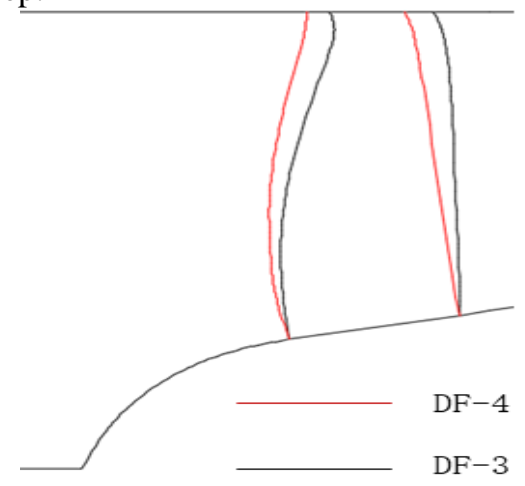

Figure 19 meridional channel comparision

As shown in Fig. 20, after being swept forward, the radial migration flow of the suction surface of the rotor is suppress, and the zone of the migration flow in the blade is reduced. Due to the forward-swept blade, the higher span of blade is exposed to the airflow earlier, so at the trailing edge, the radial pressure gradient increases[2], which is advantageous for suppressing the radial migration of the boundary layer low-energy fluid. At the same time, after the blade is swept forward, the low-energy fluid in the boundary layer will move away from the blade with the wake vortex at the lower span during the radial migration[1], avoiding the further development and accumulation of the low-energy fluid into the middle of the blade.

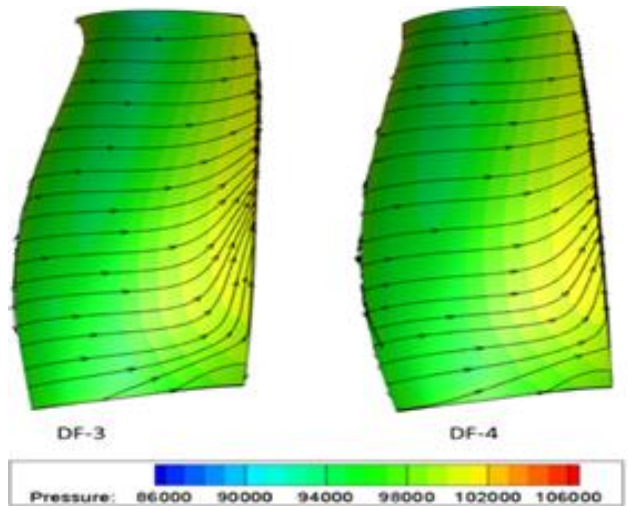

\section{Figure 20 limiting streamline of suction surface}

After the fan rotor is swept forward, the pressure ratio and efficiency characteristics of the fan are shown in Figure 21 and Figure 22. Compared with DF-3, the DF-4 has a constant pressure ratio at the large mass flow, while the efficiency increases, and at small mass flow, the pressure ratio and efficiency are significantly improved. Although the near stall mass flow is basically unchanged, the pressure ratio is increased so the margin is increased.

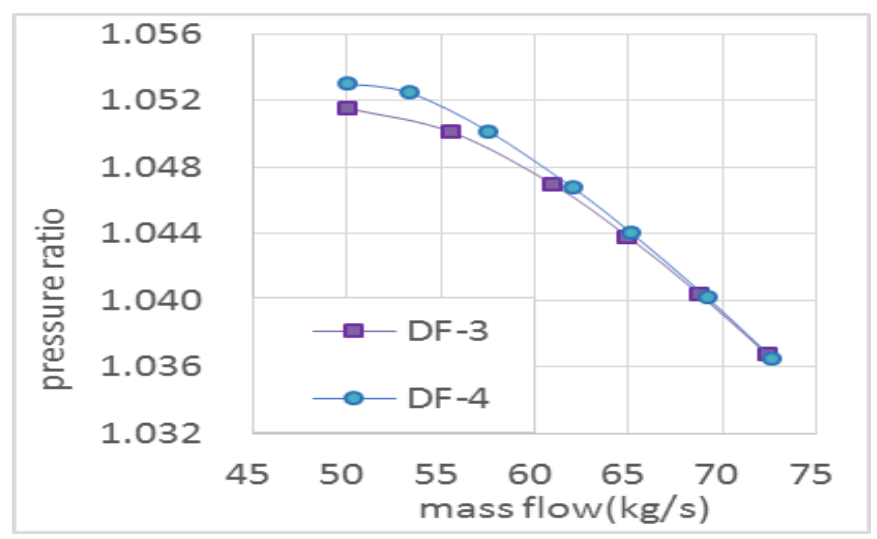

Figure 21 pressure ratio characteristic

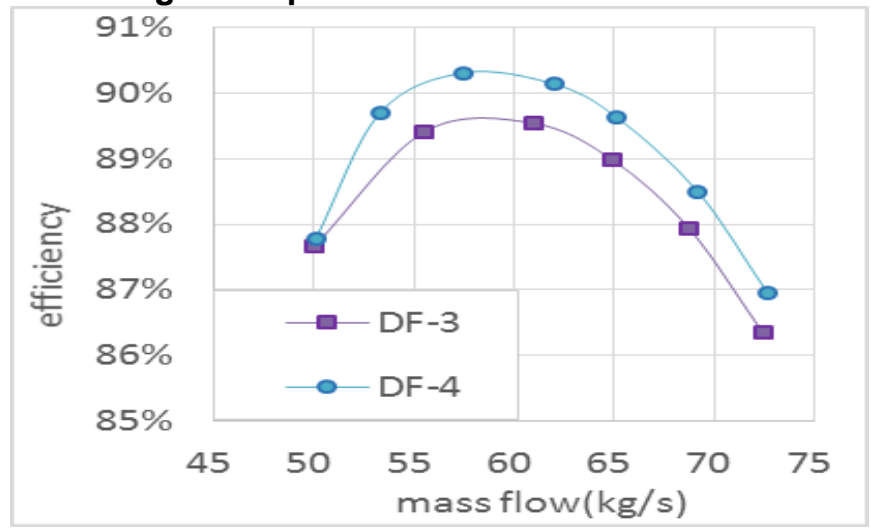

Figure 22 efficiency characteristic

\section{CONCLUSIONS}

In this paper, the influence of the load distribution along the chord direction and the span direction on the radial migration flow of the suction surface of a rotor is explored, and the influence of the forward swept blade on the radial migration flow is also explored, and the pressure ratio characteristics and efficiency characteristic of several schemes are compared.

In this paper, it is found that for a type of rotor whose roots are over-bending, when the root load is increased, the static pressure rise coefficient of the root is lowered, so that the pressure gradient from the root to the tip is increased, thereby suppressing the radial migration flow of the root.

For the radial migration flow at the middle span near the trailing edge, the load distribution along the chord direction of the region can be adjusted, the rear load can be appropriately reduced, so the front load is increased to guarantee the total load keep constant. And The pressure gradient between the suction surface and the pressure surface at the trailing edge is reduced, so the secondary flow from the pressure surface to the suction surface is attenuated. In this paper, the method can eliminate the backflow zone at the trailing edge and suppress the radial migration flow of the middle span.

For forward-swept blade, the higher span of the blade at the leading edge is exposed to the flow earlier, so the energy conversion causes the pressure of tip to rise, changing the 
original radial pressure gradient, causing the fluid to move downward to match the new pressure gradient.At the same time, when the rotor blade is swept forward, the radial migration fluid will fall off the blade surface with the wake vortex before reaching the blade tip, so the radial migration is suppressed

When the secondary flow is controlled, the pressure ratio and efficiency of the rotor will increase.

References

[1]H.Hayami,Y.Senoo, Y.I.Hyun and M.Yamaguchi,19 90,"Effects of Tip Clearance of Nozzle Vanes on Perfor mance of Radial Turbine Rotor."presented at Journal of Turbomachinery.

[2]Yamaguchi,N,Tominaga,T,Hattori,S, and Mitsuhashi, T,1991,"Secondary-Loss Reduction by Forward-Skewing of Axial Compressor Rotor Blading,"presented at Yokoh ama International Gas Turbine Conference.

[3]Patterson,D.J.and Hoeger,M.,1986,"The effect of Reynolds Number and Velocity Distribution on LP Turbine Cascade Performance,"ASME paper 86-GT-271

[4]Hashimoto,K.and Kimura,T., 1984,"Preliminary Study on Forward Loaded Cascades Designed with Inverse Method for Low Pressure Turbine,"ASME paper 84-GT-65

[5]Hoheisel,H.,Kiock,R.,Lichtfuss,H.J.,and Fottner,L.,1 987, "Influence of Free-Stream Turbulence and Blade Pre ssure Gradient on Boundary Layer and Loss Behavior of Turbine Cascades,"ASME Transactions,Journal of Turbo machinery, 109,pp.210-219

[6]Benjamin T.,Luymes, Qiang An and Adam M.Steinberg,2018,"Influence of Blade Loading Profile on Wake Dynamics in High-pressure Turbine Cascades,"ASME Transactions,Journal of Turbomachinery,210,pp.106-115.

[7]Jürgen Hübner and Leonhard Fottner, 1996,"Influence of Tip-clearance,Aspect Ratio,Blade Loading and Inlet Boundary Layer on Secondary Losses in Compressor Cascades,"ASME paper 96-GT-505.

[8]Aniwat Tiralap,Choon S.Tan and Eric Donahoo,"Effects of Rotor Tip Blade Loading Variation on Compressor Stage Performance,"ASME Transactions,Journal of Turbomachinery,139.pp.25-35.

[9]Jin Donghai,Gui Xingmin,"Numerical Simulation of Aerodynamic Performance of a Multi-stage High-load Fan/Compressor for a Turbofan Engine."Journal of Aeronautical Power,2011,26(5):1059-1065.

[10]Eric A.Crosh,Charles W.Haldeman and Michael G.Dunn ea al."Investigation of Turbine Shroud Distortions on the Aerodynamics of a One and One-Half Stage Highpressure Turbine.” ASME paper GT-2009-59562.

[11]Laroslav Druzhinin,Victor Mileshin and Vladimir Korzhnev,"Numerical and Experimental Investigation of Aerodynamic Characteristics of Model Ultra High Bypass Ratio Counter Rotating Fan.”ASME paper GT2018-76861. 